\title{
PA-013 COMPARISON OF AUTOMATIC AND MANUAL MEASUREMENT OF QT AND QTC INTERVALS DURING A CLINICAL PHASE III-B/ IV IN KOLLE, MALI
}

François Dao, Nianwalou Dara, Bouran Sidibe, Moctar Coulibaly, Allaye Tolo, Hamma Maiga, Nouhoum Ouologuem, Hamidou Niangaly, Issaka Sagara, Abdoulaye Djimde. MRTC, University of Bamako, Mali

10.1136/bmjgh-2016-000260.51

Background The detailed assessment of the QT and corrected QT (QTc) intervals prolongation is recommended when testing new drugs. The electrocardiograph automatically displays generally reliable values of the QT interval and corrected QT but morphological variations of the $\mathrm{T}$ wave may cause reading errors, hence the use of the manual measurement as an alternative method. Our objective was to evaluate the correlation between the automatic and manual measurement of QT values. Methods In Kolle from March 2012 to December 2015, an open randomised, phase III-b/IV study comparing dihydroartemisinine-piperaquine, pyronaridine-artesunate and artemether-lumefantrine was conducted. An electrocardiograph cartridge 12 electrodes coupled to a computer with the Tele Touch software was used for the electrocardiogram on Day 0 before the study drugs administration and on Day 2, 2-4 hours after the administration of the last dose of the antimalarial. The manual measurement of QT and QTc was made using the Bazett method [QTcB_m $=($ Number leaded $\times 0.04 \times \mathrm{QTcF}) / \mathrm{QTcB}]$. For prolonged QTc cases on Day 2, another measurement was done during the next scheduled visit (Days 7, 14, 21, 28, 35 and 42) until the QTc normalisation. 
Results A total of 764 ECG was recorded with 398 participants. Different automatic and manual values of QT and QTc are scattered around different medium. Comparisons of different values of QT $(p=0.1245)$ and QTc $(p<0.001)$ showed a statistically significant differences and the concordance between automatic and manual tests was QT: Rho_c $=0.77$ and QTc: Rho_c $=0.46$.

Conclusions Our results indicate no perfect match between automatic and manual methods for QT and QTc. Manual reading remains important to correct any machine errors during clinical studies. 